\title{
ON THE RANK OF SUZUKI POLYTOPES: AN ANSWER TO HUBARD AND LEEMANS
}

\author{
PABLO SPIGA
}

\begin{abstract}
In this paper we show that the rank of every chiral polytope having a Suzuki group as automorphism group is 3. This gives a positive answer to a conjecture of Isabel Hubard and Dimitri Leemans.
\end{abstract}

\section{INTRODUCTION}

In [1, Isabel Hubard and Dimitri Leemans have embarked in an extensive analysis on the abstract polytopes admitting an almost simple group with socle a Suzuki group as automorphism group. Except for an answer to [1, Conjecture 1], their analysis is very satisfactory and gives a great insight on such abstract polytopes. This his conjecture, Hubard and Leemans ask whether the rank of every chiral polytope having a Suzuki group as automorphism group is 3. In this paper, we give a positive answer to this conjecture 1

Theorem 1.1. Let $\operatorname{Sz}(q) \leq G \leq \operatorname{Aut}(\operatorname{Sz}(q))$ with $q=2^{2 e+1}$, where $e$ is a positive integer and where $\operatorname{Sz}(q)$ is the Suzuki group defined over a finite field of cardinality $q$. Then, the rank of any chiral polytope having $G$ as automorphism group is 3 .

\section{Notation}

Let $e$ be a positive integer, let $q:=2^{2 e+1}$, let $t:=2^{e}=\sqrt{2 q}$, let $\mathbb{F}_{q}$ be a finite field of cardinality $q$, let $V:=\mathbb{F}_{q}^{4}$ be the 4 -dimensional vector space over $\mathbb{F}_{4}$ consisting of column vectors and let

$$
f: V \times V \rightarrow \mathbb{F}_{q}
$$

be the alternating bilinear form whose associated matrix (with respect to the canonical basis of $V$ ) is

$$
\iota:=\left(\begin{array}{cccc}
0 & 0 & 0 & 1 \\
0 & 0 & 1 & 0 \\
0 & 1 & 0 & 0 \\
1 & 0 & 0 & 0
\end{array}\right) .
$$

We let $\operatorname{Sp}_{4}(q)$ be the symplectic group with respect to the bilinear form $f$ and we let $\operatorname{Sz}(q)$ be the Suzuki group, viewed as a subgroup of the symplectic group $\operatorname{Sp}_{4}(q)$. (Hence, $\mathrm{Sz}(q)$ is the fixed-point subgroup of a graph-field automorphism of $\left.\mathrm{Sp}_{4}(q).\right)$

We now use the characterization of Wilson [3] of the Suzuki subgroup $\operatorname{Sz}(q)$ in $\operatorname{Sp}_{4}(q)$. This characterization is via a suitable commutative product on $V$. Wilson has defined a commutative product

$$
\bullet: V \times V \rightarrow V
$$

by setting

$$
\begin{aligned}
& e_{1} \bullet e_{1}=0, \\
& e_{1} \bullet e_{2}=e_{2}, \\
& e_{1} \bullet e_{3}=e_{4}, \\
& e_{1} \bullet e_{4}=0,
\end{aligned}
$$$$
e_{2} \bullet e_{1}=e_{2} \text {, }
$$$$
e_{2} \bullet e_{2}=0 \text {, }
$$$$
e_{2} \bullet e_{3}=0
$$$$
e_{2} \bullet e_{4}=e_{1},
$$

$$
\begin{aligned}
& e_{3} \bullet e_{1}=e_{4}, \\
& e_{3} \bullet e_{2}=0, \\
& e_{3} \bullet e_{3}=0, \\
& e_{3} \bullet e_{4}=e_{3},
\end{aligned}
$$

$$
\begin{aligned}
& e_{4} \bullet e_{1}=0, \\
& e_{4} \bullet e_{2}=e_{1}, \\
& e_{4} \bullet e_{3}=e_{3}, \\
& e_{4} \bullet e_{4}=0,
\end{aligned}
$$

and by extending $\bullet$ to the whole of $V$ using the following formula:

$$
\left(\sum_{i=1}^{4} \lambda_{i} e_{i}\right) \bullet\left(\sum_{j=1}^{4} \mu_{j} e_{j}\right)=\sum_{i, j=1}^{4} \lambda_{i}^{t} \mu_{j}^{t}\left(e_{i} \bullet e_{j}\right) .
$$

2010 Mathematics Subject Classification. 52B11, $20 \mathrm{D} 06$.

Key words and phrases. abstract chiral polytopes, Suzuki simple groups.

${ }^{1}$ I first heard about this conjecture in the lovely 2014 summer course in algebraic graph theory by Dimitri Leemans in Rogla, Slovenia. Here I express my gratitude to Dimitri for such a wonderful series of lectures and to the organizers of the Rogla Summer School. 
Wilson [3] has shown that $\mathrm{Sz}(q)$ consists exactly of the matrices $g$ in $\operatorname{Sp}_{4}(q)$ (that is, the matrices preserving the bilinear form $f$ ) which satisfy

$$
g u \bullet g v=g(u \bullet v), \quad \forall u, v \in V \text { with } f(u, v)=0 .
$$

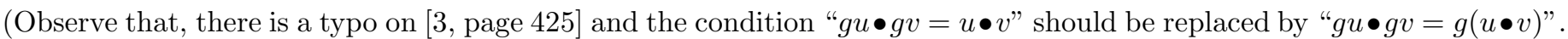
This typo has already be spotted in the mathview by Colva Roney-Dougal.) We use this characterization of Sz( $q)$ in the proof of Theorem 1.1 .

\section{Proof of Theorem 1.1}

We argue by contradiction and we suppose that there exists $G$ with $\operatorname{Sz}(q) \leq G \leq \operatorname{Aut}(\operatorname{Sz}(q))$, for some positive integer $e$, such that $G$ admits a chiral polyhedron of rank different from 3. In view of [1, Theorem 1], we may suppose that $G=\operatorname{Sz}(q)$ and that $\mathrm{Sz}(q)$ admits a chiral 4-polytope. In particular, there exist $\sigma_{1}, \sigma_{2}, \sigma_{3} \in \operatorname{Sz}(q)$ with

$$
\operatorname{Sz}(q)=\left\langle\sigma_{1}, \sigma_{2}, \sigma_{3}\right\rangle, \quad \text { where } \sigma_{1} \sigma_{2} \sigma_{3}, \sigma_{2} \sigma_{3}, \sigma_{1} \sigma_{2} \text { are involutions of } \operatorname{Sz}(q) \text {. }
$$

Since the involutions in $\mathrm{Sz}(q)$ form a unique conjugacy class [2] and since $\iota \in \mathrm{Sz}(q)$, we may suppose that

$$
\iota=\sigma_{1} \sigma_{2} \sigma_{3} .
$$

Since $\sigma_{1} \sigma_{2}=\iota \sigma_{3}^{-1}$ is an involution, we deduce $1=\left(\iota \sigma_{3}^{-1}\right)^{2}=\iota \sigma_{3}^{-1} \iota \sigma_{3}^{-1}$ and hence $\sigma_{3}^{-1} \iota \sigma_{3}^{-1}=\iota$. An entirely similar computation yields $\sigma_{1}^{-1} \iota \sigma_{1}^{-1}=\iota$. Thus

$$
\sigma_{1}^{-1}, \sigma_{3}^{-1} \in \mathcal{X}:=\{x \in \mathrm{Sz}(q) \mid x \iota x=\iota\}
$$

In what follows we denote by $x^{\perp}$ the transpose matrix of $x$. As $\operatorname{Sz}(q) \leq \operatorname{Sp}_{4}(q)$ and as $\iota$ is the matrix associated to the bilinear form $f$, we have $x^{\perp} \iota x=\iota$, for every $x \in \operatorname{Sz}(q)$. In particular, for every $x \in \mathcal{X}$, we have $x^{\perp} \iota x=x \iota x$ and hence

$$
x^{\perp}=x .
$$

In particular, $x$ is a symmetric matrix and hence we may write

$$
x=\left(\begin{array}{cccc}
a_{11} & a_{12} & a_{13} & a_{14} \\
a_{12} & a_{22} & a_{23} & a_{24} \\
a_{13} & a_{23} & a_{33} & a_{34} \\
a_{14} & a_{24} & a_{34} & a_{44}
\end{array}\right) .
$$

We use the characterization of Wilson to determine when a symmetric matrix $x$ as in (2) lies in $\mathrm{Sz}(q)$.

From

$$
\begin{aligned}
& x e_{1}=a_{11} e_{1}+a_{12} e_{2}+a_{13} e_{3}+a_{14} e_{4}, \\
& x e_{2}=a_{12} e_{1}+a_{22} e_{2}+a_{23} e_{3}+a_{24} e_{4}, \\
& x e_{3}=a_{13} e_{1}+a_{23} e_{2}+a_{33} e_{3}+a_{34} e_{4}, \\
& x e_{4}=a_{14} e_{1}+a_{24} e_{2}+a_{34} e_{3}+a_{44} e_{4},
\end{aligned}
$$

we deduce

$$
\begin{aligned}
x e_{2}= & x\left(e_{1} \bullet e_{2}\right)=x e_{1} \bullet x e_{2}=\left(a_{11} e_{1}+a_{12} e_{2}+a_{13} e_{3}+a_{14} e_{4}\right) \bullet\left(a_{12} e_{1}+a_{22} e_{2}+a_{23} e_{3}+a_{24} e_{4}\right), \\
= & a_{11}^{t} a_{12}^{t}\left(e_{1} \bullet e_{1}\right)+a_{11}^{t} a_{22}^{t}\left(e_{1} \bullet e_{2}\right)+a_{11}^{t} a_{23}^{t}\left(e_{1} \bullet e_{3}\right)+a_{11}^{t} a_{24}^{t}\left(e_{1} \bullet e_{4}\right) \\
& +a_{12}^{t} a_{12}^{t}\left(e_{2} \bullet e_{1}\right)+a_{12}^{t} a_{22}^{t}\left(e_{2} \bullet e_{2}\right)+a_{12}^{t} a_{23}^{t}\left(e_{2} \bullet e_{3}\right)+a_{12}^{t} a_{24}^{t}\left(e_{2} \bullet e_{4}\right) \\
& +a_{13}^{t} a_{12}^{t}\left(e_{3} \bullet e_{1}\right)+a_{13}^{t} a_{22}^{t}\left(e_{3} \bullet e_{2}\right)+a_{13}^{t} a_{23}^{t}\left(e_{3} \bullet e_{3}\right)+a_{13}^{t} a_{24}^{t}\left(e_{3} \bullet e_{4}\right) \\
& +a_{14}^{t} a_{12}^{t}\left(e_{4} \bullet e_{1}\right)+a_{14}^{t} a_{22}^{t}\left(e_{4} \bullet e_{2}\right)+a_{14}^{t} a_{23}^{t}\left(e_{4} \bullet e_{3}\right)+a_{14}^{t} a_{24}^{t}\left(e_{4} \bullet e_{4}\right) \\
= & a_{11}^{t} a_{22}^{t}\left(e_{1} \bullet e_{2}\right)+a_{11}^{t} a_{23}^{t}\left(e_{1} \bullet e_{3}\right)+a_{12}^{t} a_{12}^{t}\left(e_{2} \bullet e_{1}\right)+a_{12}^{t} a_{24}^{t}\left(e_{2} \bullet e_{4}\right) \\
& +a_{13}^{t} a_{12}^{t}\left(e_{3} \bullet e_{1}\right)+a_{13}^{t} a_{24}^{t}\left(e_{3} \bullet e_{4}\right)+a_{14}^{t} a_{22}^{t}\left(e_{4} \bullet e_{2}\right)+a_{14}^{t} a_{23}^{t}\left(e_{4} \bullet e_{3}\right) \\
= & a_{11}^{t} a_{22}^{t} e_{2}+a_{11}^{t} a_{23}^{t} e_{4}+a_{12}^{t} a_{12}^{t} e_{2}+a_{12}^{t} a_{24}^{t} e_{1}+a_{13}^{t} a_{12}^{t} e_{4}+a_{13}^{t} a_{24}^{t} e_{3}+a_{14}^{t} a_{22}^{t} e_{1}+a_{14}^{t} a_{23}^{t} e_{3} \\
= & \left(a_{12}^{t} a_{24}^{t}+a_{14}^{t} a_{22}^{t}\right) e_{1}+\left(a_{11}^{t} a_{22}^{t}+a_{12}^{t} a_{12}^{t}\right) e_{2}+\left(a_{13}^{t} a_{24}^{t}+a_{14}^{t} a_{23}^{t}\right) e_{3}+\left(a_{11}^{t} a_{23}^{t}+a_{13}^{t} a_{12}^{t}\right) e_{4}
\end{aligned}
$$

Therefore we obtain the system of equations:

$$
\begin{aligned}
a_{12}^{t} a_{24}^{t}+a_{14}^{t} a_{22}^{t} & =a_{12}, \\
a_{11}^{t} a_{22}^{t}+a_{12}^{2 t} & =a_{22}, \\
a_{13}^{t} a_{24}^{t}+a_{14}^{t} a_{23}^{t} & =a_{23}, \\
a_{11}^{t} a_{23}^{t}+a_{13}^{t} a_{12}^{t} & =a_{24}
\end{aligned}
$$


An entirely analogous computation can be done imposing

$$
\begin{aligned}
& x e_{1} \bullet x e_{3}=x\left(e_{1} \bullet e_{3}\right)=x e_{4}, \quad x e_{4} \bullet x e_{3}=x\left(e_{4} \bullet e_{3}\right)=x e_{3}, x e_{1} \bullet x e_{3}=x\left(e_{2} \bullet e_{4}\right)=x e_{1}, \\
& x e_{1} \bullet x e_{4}=x\left(e_{1} \bullet e_{4}\right)=x \cdot 0=0, x e_{2} \bullet x e_{3}=x\left(e_{2} \bullet e_{3}\right)=x \cdot 0=0 .
\end{aligned}
$$

We skip the computations above and we report the resulting equations:

$$
\begin{aligned}
a_{12}^{t} a_{34}^{t}+a_{14}^{t} a_{23}^{t} & =a_{14}, \\
a_{13}^{t} a_{34}^{t}+a_{14}^{t} a_{33}^{t} & =a_{34}, \\
a_{11}^{t} a_{33}^{t}+a_{13}^{2 t} & =a_{44} \\
a_{22}^{t} a_{44}^{t}+a_{24}^{2 t} & =a_{11}, \\
a_{23}^{t} a_{44}^{t}+a_{24}^{t} a_{34}^{t} & =a_{13}, \\
a_{33}^{t} a_{44}^{t}+a_{34}^{2 t} & =a_{33}, \\
a_{14} a_{24} & =a_{12} a_{44}, \\
a_{12} a_{14} & =a_{11} a_{24}, \\
a_{13} a_{44} & =a_{14} a_{34}, \\
a_{11} a_{34} & =a_{13} a_{14}, \\
a_{22} a_{34} & =a_{24} a_{23}, \\
a_{22} a_{13} & =a_{12} a_{23}, \\
a_{23} a_{34} & =a_{24} a_{33}, \\
a_{12} a_{33} & =a_{13} a_{23} .
\end{aligned}
$$

Writing the equality $x \iota x=\iota$ explicitly, we obtain

$$
\begin{aligned}
\left(\begin{array}{llll}
0 & 0 & 0 & 1 \\
0 & 0 & 1 & 0 \\
0 & 1 & 0 & 0 \\
1 & 0 & 0 & 0
\end{array}\right) & =\left(\begin{array}{llll}
a_{11} & a_{12} & a_{13} & a_{14} \\
a_{12} & a_{22} & a_{23} & a_{24} \\
a_{13} & a_{23} & a_{33} & a_{34} \\
a_{14} & a_{24} & a_{34} & a_{44}
\end{array}\right)\left(\begin{array}{llll}
0 & 0 & 0 & 1 \\
0 & 0 & 1 & 0 \\
0 & 1 & 0 & 0 \\
1 & 0 & 0 & 0
\end{array}\right)\left(\begin{array}{llll}
a_{11} & a_{12} & a_{13} & a_{14} \\
a_{12} & a_{22} & a_{23} & a_{24} \\
a_{13} & a_{23} & a_{33} & a_{34} \\
a_{14} & a_{24} & a_{34} & a_{44}
\end{array}\right) \\
& =\left(\begin{array}{lllll}
a_{14} & a_{13} & a_{12} & a_{11} \\
a_{24} & a_{23} & a_{22} & a_{12} \\
a_{34} & a_{33} & a_{23} & a_{13} \\
a_{44} & a_{34} & a_{24} & a_{14}
\end{array}\right)\left(\begin{array}{llll}
a_{11} & a_{12} & a_{13} & a_{14} \\
a_{12} & a_{22} & a_{23} & a_{24} \\
a_{13} & a_{23} & a_{33} & a_{34} \\
a_{14} & a_{24} & a_{34} & a_{44}
\end{array}\right) .
\end{aligned}
$$

By computing explicitly the matrix product on the right hand side and by comparing the coefficients in position $(1,4)$ and $(2,3)$ with the matrix on the left hand side, we obtain two further equations

$$
\begin{aligned}
& a_{14}^{2}+a_{13} a_{24}+a_{12} a_{34}+a_{11} a_{44}=1, \\
& a_{24} a_{13}+a_{23}^{2}+a_{22} a_{33}+a_{12} a_{34}=1 .
\end{aligned}
$$

We now start solving this system of equations by dividing our task in various cases.

3.1. Case $a_{24} \neq 0$. From (13), (14), (17) and (19), we deduce

$$
a_{14}=\frac{a_{12} a_{44}}{a_{24}}, a_{11}=\frac{a_{12}^{2} a_{44}}{a_{24}^{2}}, a_{23}=\frac{a_{22} a_{34}}{a_{24}}, a_{33}=\frac{a_{22} a_{34}^{2}}{a_{24}^{2}} .
$$

Using this value of $a_{14}$ and $a_{11}$ in (21), we obtain

$$
a_{13} a_{24}+a_{12} a_{34}=1 .
$$

From (15), we get

$$
a_{13} a_{44}=\frac{a_{12} a_{44} a_{34}}{a_{24}} .
$$

Using this equality and multiplying (24) by $a_{44} / a_{24}$, we deduce

$$
\frac{a_{44}}{a_{24}}=\frac{a_{44}}{a_{24}}\left(a_{13} a_{24}+a_{12} a_{34}\right)=a_{13} a_{44}+\frac{a_{12} a_{44} a_{34}}{a_{24}}=0 .
$$

Therefore $a_{44}=0$. Using this value of $a_{44}$ in (23), we deduce $a_{14}=a_{11}=0$.

Substituting the value of $a_{23}$ from (23) in (18), we get $a_{22} a_{13}=\frac{a_{12} a_{22} a_{34}}{a_{24}}$. Therefore, multiplying (24) by $a_{22} / a_{24}$, we obtain

$$
\frac{a_{22}}{a_{24}}=\frac{a_{22}}{a_{24}}\left(a_{13} a_{24}+a_{12} a_{34}\right)=a_{22} a_{13}+\frac{a_{12} a_{22} a_{34}}{a_{24}}=0 .
$$


Therefore $a_{22}=0$. Using this value of $a_{22}$ in (44), we deduce $a_{12}=0$. Now, (23) gives $a_{33}=0$. From (9), we get $a_{13}=0$. Summing up,

$$
a_{11}=a_{12}=a_{13}=a_{14}=0,
$$

contradicting the fact that $x$ is a non-singular matrix.

3.2. Case $a_{24}=0$ and $a_{12} \neq 0$. From (13), (14), we obtain

$$
a_{44}=0, a_{14}=0 .
$$

Now, (21) gives $a_{12} a_{34}=1$. However, (3) with $a_{24}=a_{14}=0$ gives $a_{12}=0$, which is a contradiction.

3.3. Case $a_{24}=a_{12}=0$ and $a_{34} \neq 0$. From (17), we have $a_{22}=0$. Using this, from (22), we get $a_{23}=1$. However, (19) yields $a_{23} a_{34}=0$, which is a contradiction.

3.4. Case $a_{24}=a_{12}=a_{34}=0$ and $a_{13} \neq 0$. From (15), we have $a_{44}=0$. Now, (21) yields $a_{14}=1$. However, (16) gives $a_{13} a_{14}=0$, which is a contradiction.

3.5. Case $a_{24}=a_{12}=a_{34}=a_{13}=0$ and $a_{14} \neq 0$. From (3), we have $a_{22}=0$. Now, (22) gives $a_{23}=1$. In turn, from (6), we obtain $a_{11}=0$. Using this, from (21), we get $a_{14}=1$. From (8) and (9), we get $a_{33}=a_{44}=0$. Therefore

$$
x=\iota
$$

and we have our first element in $\mathcal{X}$.

3.6. Case $a_{24}=a_{12}=a_{34}=a_{13}=a_{14}=0$. From (21), we have $a_{44}=a_{11}^{-1}$. Now, (5) yields $a_{23}=0$. In turn, from (22), we have $a_{33}=a_{22}^{-1}$. Finally, (10) yields $a_{22}^{t} a_{11}^{-t}=a_{1} 1$, that is, $a_{22}^{t}=a_{11}^{t+1}$. Raising both sides of this equality by $2 t$ and recallying that $2 t^{2}=q$, we obtain $a_{22}=a_{11}^{2 t+1}$. Therefore

$$
x=\left(\begin{array}{cccc}
a_{11} & 0 & 0 & 0 \\
0 & a_{11}^{2 t+1} & 0 & 0 \\
0 & 0 & a_{11}^{-2 t-1} & 0 \\
0 & 0 & 0 & a_{11}^{-1}
\end{array}\right) .
$$

Summing up, we have shown that

$$
\mathcal{X}=\left\{\left(\begin{array}{llll}
0 & 0 & 0 & 1 \\
0 & 0 & 1 & 0 \\
0 & 1 & 0 & 0 \\
1 & 0 & 0 & 0
\end{array}\right)\right\} \cup\left\{\left(\begin{array}{cccc}
a & 0 & 0 & 0 \\
0 & a^{2 t+1} & 0 & 0 \\
0 & 0 & a^{-2 t-1} & 0 \\
0 & 0 & 0 & a^{-1}
\end{array}\right) \mid a \in \mathbb{F}_{q} \backslash\{0\}\right\} .
$$

3.7. Pulling the threads of the argument. We now use (1). If $\sigma_{1}^{-1}$ or $\sigma_{3}^{-1}$ equals $\iota$, then we obtain that either $\sigma_{1} \sigma_{2}=1$ or $\sigma_{2} \sigma_{3}=1$, contradicting the fact that $\sigma_{1} \sigma_{2}$ and $\sigma_{2} \sigma_{3}$ are both involutions. Therefore,

$$
\begin{aligned}
\sigma_{1} & =\left(\begin{array}{cccc}
a^{-1} & 0 & 0 & 0 \\
0 & a^{-2 t-1} & 0 & 0 \\
0 & 0 & a^{2 t+1} & 0 \\
0 & 0 & 0 & a
\end{array}\right) \\
\sigma_{3} & =\left(\begin{array}{cccc}
b^{-1} & 0 & 0 & 0 \\
0 & b^{-2 t-1} & 0 & 0 \\
0 & 0 & b^{2 t+1} & 0 \\
0 & 0 & 0 & b
\end{array}\right),
\end{aligned}
$$

for some $a, b \in \mathbb{F}_{q} \backslash\{0\}$. Observe that $\sigma_{1}$ and $\sigma_{3}$ centralize each other. In particular, $\left\langle\sigma_{1}, \sigma_{3}\right\rangle$ is abelian. Now,

$$
\operatorname{Sz}(q)=\left\langle\sigma_{1}, \sigma_{2}, \sigma_{3}\right\rangle=\left\langle\sigma_{1}, \sigma_{3}, \sigma_{1} \sigma_{2} \sigma_{3}\right\rangle=\left\langle\sigma_{1}, \sigma_{3}, \iota\right\rangle .
$$

An elementary computation gives $\sigma_{1}^{\iota}=\sigma_{1}^{-1}$ and $\sigma_{3}^{\iota}=\sigma_{3}^{-1}$. Therefore, $\left\langle\sigma_{1}, \iota, \sigma_{3}\right\rangle$ is soluble. As $\mathrm{Sz}(q)$ is a non-abelian simple group, we obtain our final contradiction.

\section{REFERENCES}

[1] I. Hubard, D. Leemans, Chiral polytopes and Suzuki simple groups. Rigidity and symmetry, 155-175, Fields Inst. Commun. 70, Springer, New York, 2014

[2] M. Suzuki, On a class of doubly transitive groups, Ann. Math. 75, 105-145, 1962.

[3] R. A. Wilson, A new approach to the Suzuki groups, Math. Proc. Cambridge Philos. Soc. 148 (2010), 425-428.

Pablo Spiga, Dipartimento di Matematica e Applicazioni, University of Milano-Bicocca,

Via Cozzi 55, 20125 Milano, Italy

Email address: pablo.spiga@unimib.it 